\title{
The design and pilot of a translation aid to help ED clinicians enhance communication with the Portuguese-speaking patient
}

\author{
Alice Han • Humberto Laranjo • Steven M. Friedman
}

Received: 7 May 2008 / Accepted: 27 December 2008 /Published online: 24 February 2009

(C) Springer-Verlag London Ltd 2009

\begin{abstract}
Background Language barriers hinder health care delivery in settings with culturally diverse populations. Interventions to accommodate non-English-speaking patients have been shown to shorten length of stay and reduce non-urgent visits.

Aims Our aim was to design and do a pilot study on an instrument to facilitate history taking with Portuguesespeaking patients in the emergency department (ED).

Methods An instrument was designed to facilitate history taking with Portuguese-speaking patients (PSPs). A pocketsized document incorporated, bilingual, problem-oriented, closed-ended questions for common ED presentations as well as numbers, measurements of time, and anatomy. A paired audio tutorial on a compact disk (CD) demonstrated correct pronunciation of each phrase. A 3-month pilot was undertaken in a downtown teaching hospital on a convenience sample of PSPs who indicated the need for a translator at triage. A trained Portuguese-speaking observer
\end{abstract}

A. Han $\cdot$ S. M. Friedman

Faculty of Medicine, University of Toronto and Dalla Lana

School of Public Health, University of Toronto,

Toronto, ON, Canada

A. Han

e-mail: a.han@utoronto.ca

H. Laranjo

University Health Network,

Toronto, ON, Canada

e-mail: hlaranjo@yorku.ca

S. M. Friedman $(\square)$

RFE G-S434, Toronto General Hospital,

200 Elizabeth Street,

Toronto, ON M5G 2C4, Canada

e-mail: steven.friedman@uhn.on.ca monitored clinician/patient pairs using the instrument and scored differential patient comprehension in a standardized manner. Qualitative patient and clinician impressions were assessed. A follow-up survey assessed emergency physician (EP) impressions of the instrument.

Results Eight of nine eligible clinician/patient pairs were enrolled. The average proportions of questions answered appropriately in English and then using the instrument were $16.7 \%$ and $85.5 \%$, respectively, with mean improvement of $68.8 \%$ (confidence interval: 45.6-92.1). Most (7/8) patients agreed that the instrument had helped in communication. Half (4/8) of the clinicians indicated that the tool had helped them communicate, and most (7/8) indicated that they would use the instrument in the future. Few $(2 / 17)$ physicians utilized the audio guide. Suggested modifications included incorporation of phonetics.

Conclusions The pilot of the instrument was well received by patients and resulted in improved communication.

Keywords Emergency· Language .

Communication barriers · Translation · Portuguese

\section{Introduction}

Language barriers (LBs) hinder health care delivery in settings with culturally diverse populations. LBs decrease access to primary and preventive care [1], patient comprehension, and patient satisfaction [2]. The implications of LBs may be greatest in the emergency department (ED). Often considered the "safety net" for the health care system, EDs are used disproportionately by non-English speakers facing barriers to conventional forms of primary care within the health care system [3-8]. ED care is characterized by brief encounters between health care staff and patients [9] and 
is increasingly pressured by ED overcrowding [10], increased patient volumes [11], and relative understaffing, all of which hamper optimal physician-patient communication.

Non-English-speaking patients have cited language as the single greatest barrier to health care in the ED [12]. LBs have been reported to increase ED visit times [13], impede diagnoses [14], and possibly lead to inappropriate medication and hospitalization [12]. They may decrease patient compliance upon discharge from the ED and lower comprehension levels of discharge instructions $[15,16]$. Interventions to accommodate non-English-speaking patients have been shown to shorten length of stay and reduced non-urgent visits [17].

The handful of ED interventions employed to bridge LBs all have limitations. Cost constraints often limit the availability of in-house language interpreters to weekday business hours. Patients are often less satisfied with such intermediaries than when speaking directly to the physician, and training physicians in communication skills has been advised [18]. Telephone translation services are effective, but introduce a third party, and can be costly. Websites and computer programs that translate common medical vocabulary are frequently cumbersome to use $[19,20]$. Hospital employees and family members serving as unskilled translators compromise patient confidentiality and raise potential for medical misunderstanding from using an unskilled translator, who may be reluctant to relay bad news or embarrassing questions to the patient [21].

We designed and pilot tested a brief language education and translation aid for physicians in the ED, as a practical and economical adjunct to currently available strategies. The primary objective of this pilot study was to assess whether the ability of the ED clinician to speak limited Portuguese could enhance emergency physician (EP) ability to obtain a directed medical history among non-English-speaking Portuguese patients. A secondary objective was to investigate clinician and patient perceptions of this intervention. This study represents the first of its kind within Canada, and the first of its kind in Portuguese in North America.

\section{Methodology and methods}

Study design The protocol was piloted over a 3-month period in the ED of Toronto Western Hospital, University Health Network, a quaternary care inner city teaching hospital with 42,000 ED visits per year. The hospital is situated in an ethnically diverse community with immigrants from Portugal, China, and Latin America, and approximately $49 \%$ of ED patients speak a language other that English as a first language. Portuguese is the most common non-English first language (17\% of ED patients) [22].
The study protocol was approved by the hospital Research Ethics Board. All consent forms for patients were available in Portuguese, and a Portuguese-speaking research assistant was available to assist if the patient was illiterate.

Inclusion criteria Patients eligible for recruitment were those over the age of 17 who registered in the ED as a Portuguese-speaking patient (PSP) and indicated requiring an interpreter. Patients were excluded if they required urgent treatment, had a decreased level of consciousness, were intoxicated or otherwise unable to provide informed consent, or were in police custody. ED clinicians were eligible (EPs, nurse practitioners, residents, and senior medical students) if they were working in the ED and did not speak Portuguese.

The protocol consisted of (1) development of a paperbased, problem-oriented language translation aid and companion audio compact disk (CD) demonstrating proper pronunciation of the text in Portuguese, (2) distribution of the instrument and audio $\mathrm{CD}$ to ED clinicians for home practice, (3) piloting the instrument at bedside, with postapplication survey of patient and clinician, and (4) post-pilot evaluation survey to assess EP impressions of the instrument.

Language education instrument The language instrument consisted of two components: the paper translation instrument and the CD audio guide. (A) Paper instrument: A pocket-sized, foldable brochure (Figs. 1 and 2) was designed to facilitate taking a problem-based history with PSPs in the ED. All phrases were printed as Portuguese and English pairs and employed closed-ended questions designed to prompt responses that were clinically meaningful to the non-Portuguese-speaking questioner, i.e., with yes/no answers, a numeric response, or pointing to a region. Questions and content were formulated by an ED physician (SMF), reviewed by two independent EPs for face and content validity, and translated by a bilingual, native Portuguese-speaking ED nurse (HL). The first column of the instrument translated basic greetings. Each subsequent column facilitated a functional inquiry and history for a common system complaint in the ED, including: cardiac, respiratory, abdominal complaints/genitourinary/gastrointestinal, laceration/minor injury, and headache. An additional column translated anatomy and measurements of time (numbers, telling the time, information to specify dates, such as the days of the week, months, and years).

(B) Audio guide: The purpose of the audio guide was to reinforce proper Portuguese pronunciation. Audio clips were created for each Portuguese phrase by a native Portuguese-speaking ED nurse (HL). The CD audio guide graphically reproduced the pocket brochure, with "icons" alongside the text that would play the spoken Portuguese text upon a mouse click. 


\begin{tabular}{|c|c|c|c|c|}
\hline $\begin{array}{l}\text { Anatomy } \\
\text { Head } \\
\text { Eye } \\
\text { Nose } \\
\text { Mouth } \\
\text { Tongue } \\
\text { Ear } \\
\text { Neck } \\
\text { Shoulder, } \\
\text { Arum } \\
\text { Elbow } \\
\text { Wrist } \\
\text { Finger } \\
\text { Back } \\
\text { Spine } \\
\text { Chest } \\
\text { Heart } \\
\text { Lung } \\
\text { Liver } \\
\text { Stomach } \\
\text { Leg } \\
\text { Thigh } \\
\text { Calf } \\
\text { Knee } \\
\text { Ankle } \\
\text { Toe } \\
\text { Joint } \\
\text { Skin } \\
\text { Bone } \\
\text { Blood } \\
\text { Trpes of inj } \\
\text { Cut } \\
\text { Bruise } \\
\text { Fracture }\end{array}$ & 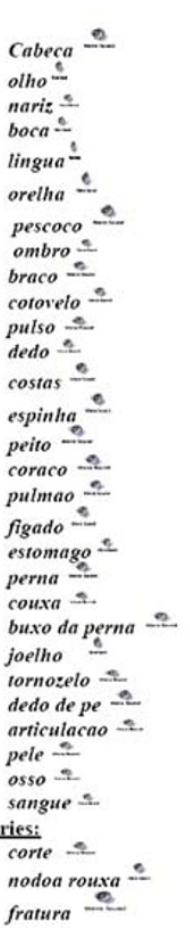 & 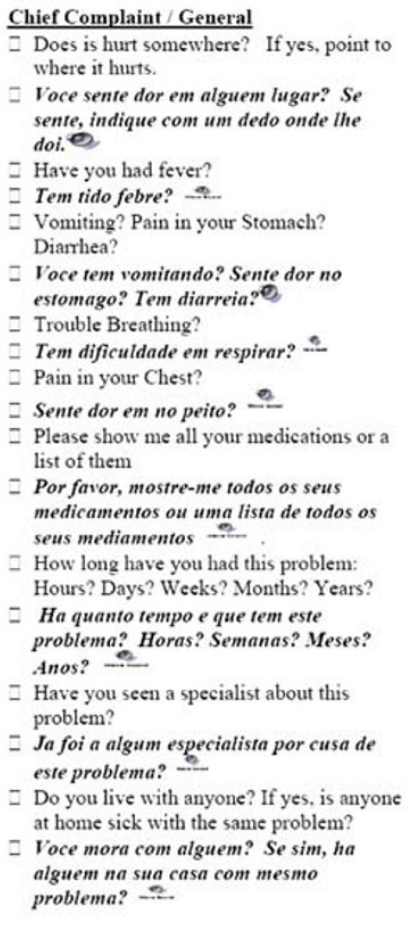 & 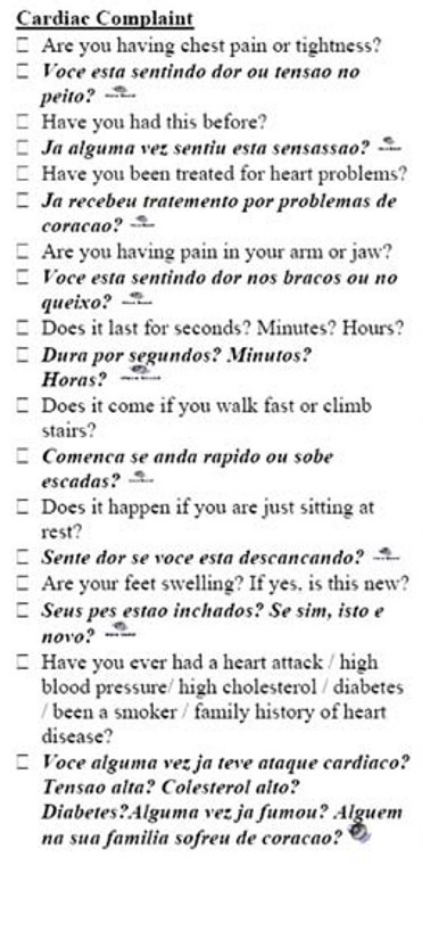 & 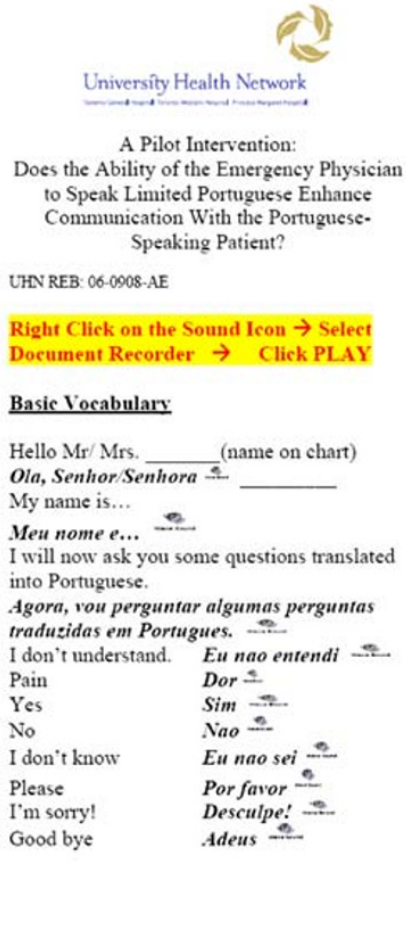 \\
\hline
\end{tabular}

*(Microphone Icons appear on CD version and play Portuguese text upon mouse click)

Fig. 1 Front: reference card for English/Portuguese problem-oriented ED history.* (Microphone icons appear on CD version and play Portuguese text upon mouse click)

The CD was placed in the ED mailbox of each EP $(n=$ 30) and over a 3-week period, EPs were sent regular weekly e-mail reminders, along with an electronic copy of the paper instrument, describing the upcoming pilot and encouraging review of the instrument and audio guide. Though clinicians were encouraged to orient themselves to the CD contents, failure to do so would not preclude study participation.

Bedside piloting and scoring A convenience sample of approximately 15 piloting shifts, determined by the researcher/observer $(\mathrm{AH})$, covered a distribution of days, evening, nights, weekdays, and weekends. Eligible patients and their attending clinicians were identified by the ED triage nurse, ED charge nurse, and ED clinician, upon being informed by the researcher that piloting was occurring for the next period of 4-6 h.

During data collection shifts, a trained Portuguesespeaking researcher $(\mathrm{AH})$ recruited eligible patients and their clinicians for informed consent and enrollment in the study. Participating clinicians were instructed to use the instrument for obtaining a history from the patient. They could select any questions they chose from the printed instrument, but were to ask each question first in English and then in Portuguese. (Each patient thus served as his or her own control.) The observer scored the response to each question (first in English, then in Portuguese) as to whether it yielded a response that was appropriate for the question asked and comprehensible to the non-Portuguese-speaking clinician. (For example, for the question "Are you having chest pain?" an indication in the positive or affirmative would be deemed appropriate; a response that did not speak directly to the question, i.e., "I'm short of breath" or "It's okay" would not be regarded as appropriate). Physicians were permitted to terminate use of the instrument at their discretion. After completion of history taking, both patient and physician were interviewed in a standardized manner regarding the intervention's effectiveness in assisting with communication.

Post-pilot evaluation After termination of the data collection period, an electronic follow-up survey of all ED clinicians initially recruited for participation was undertaken over a 2-week period to further elaborate barriers to communication in the ED, EP compliance with the study protocol, and physician impressions of the instrument

Statistical analyses Statistical analysis was performed using MS Excel and Stata. A thematic content analysis was performed on responses to open-ended questions on the surveys of both patients and clinicians. To identify common words and themes, frequencies of all the words 


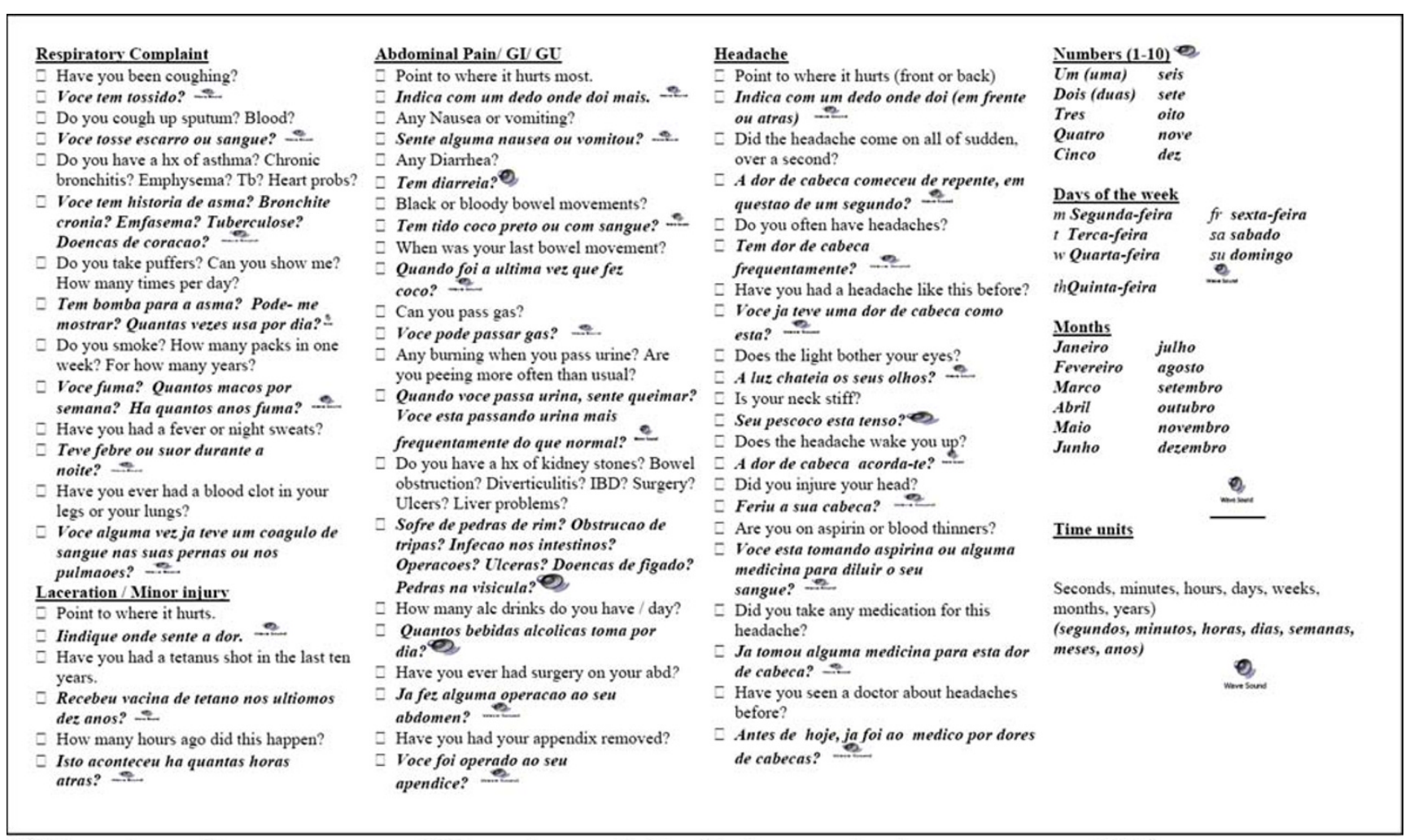

\section{*(Microphone Icons appear on CD version and play Portuguese text upon mouse click)}

Fig. 2 Rear: reference card for English/Portuguese problem-oriented ED history.* (Microphone icons appear on CD version and play Portuguese text upon mouse click)

contained in the responses to this question in the database were tabulated to identify frequently occurring words.

\section{Results}

Sampling Eleven patients were eligible to participate in the study. Two patients were excluded for reasons of mental competence (psychiatric presentation), and one patient was excluded because the attending physician declined participation in the study. Eight clinician/PSP pairs completed the pilot (five emergency physicians, one emergency department nurse practitioner, one intern, and one senior medical student.)

Effectiveness of tool The average proportions of questions answered appropriately in English and then in Portuguese were $16.7 \%$ and $85.5 \%$ respectively, with mean improvement of $68.8 \%$ (confidence interval: 45.6-92.1) (see Fig. 3).

Perceptions of tool The majority of patients (7/8) agreed or strongly agreed that the language tool helped them to communicate with the clinician. The majority of patients $(5 / 8)$ indicated approval of the tool, indicating that it was helpful $(n=2)$, made them feel more comfortable $(n=2)$, and should be expanded $(n=1)$ (see Table 1$)$.
None of the clinicians had reviewed the audio CD prior to using the language translation tool at the bedside. However, half of the clinicians (4/8) agreed or strongly agreed that the tool helped them to communicate, though two were neutral and two disagreed that the instrument would help them in the future. Most physicians (7/8) agreed or strongly agreed that they would want to use the tool in the future. Physicians expressed difficulties with Portuguese language pronunciation (Table 1).

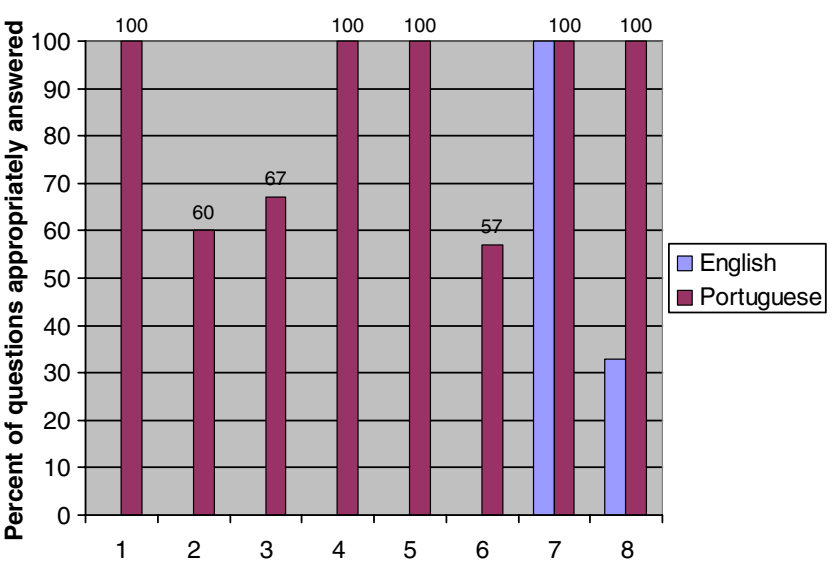

Fig. 3 Enhanced patient ability to provide medical history using language tool ( $n=8$ patients, 46 English-Portuguese language pairs) 
Table 1 Thematic analysis: patient and clinician perspectives on the instrument

Words used to identify themes expressing patient perception about the instrument $(n=5)$

Theme

Intervention is helpful

Intervention was comforting

Intervention should be expanded
Issues with pronunciation

Good tool

Cumbersome

Tool not useful because patient understood English well

\section{Keywords}

"Will help people who can't speak English."

"It made me feel good when he spoke Portuguese."

"All doctors should try to learn more than one language."
2

1

instrument $(n=7)$

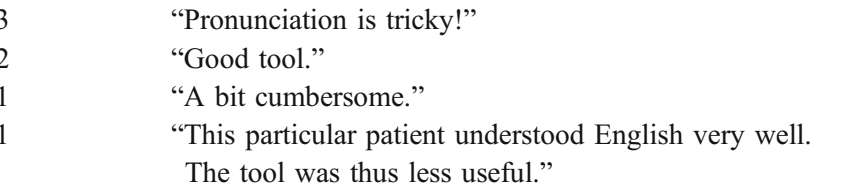

Follow-up physician survey The response rate to the poststudy survey was high ( $n=25 / 30$ surveyed). In the needs analysis component of the survey, all EPs $(n=25)$ indicated that LBs hindered communication with non-English-speaking patients, most $(23 / 25)$ felt that overcoming the Portuguese LB in particular would enhance care efforts, and few $(3 / 25)$ were satisfied with current modalities available to communicate with non-English-speaking patients.

The majority of emergency physicians $(20 / 25)$ had read the pre-study e-mails introducing the study, though only two thirds (16/25) had reviewed the attached document copy of the language translation instrument, and few $(2 / 25)$ tried the sound clips on the audio CD.

Free text clinical comments included apprehension regarding potential "false understanding" created by translation aids, a suggestion for incorporation of phonetic texts in the instrument, and an expression of dissatisfaction with the suggestion of acquiring language skills to accommodate non-English-speaking patients without their own interpreter.

\section{Discussion}

This study piloted an innovative intervention to improve communication with a subset of non-English-speaking patients in the ED. The intervention was well received by patients and physicians and resulted in improved communication despite suboptimal physician preparation. Our study supports previous literature indicating the beneficial impact of attempting cultural competency [17]. Patients were extremely appreciative of EP attempts at language competency, despite the fact that the clinician spoke only a limited number of phrases in Portuguese. This model has the potential to be utilized with other languages and is easily adaptable for use in other EDs.

Other interventions designed to bridge LBs in the ED have been described. Bischoff et al. utilized brief training aimed at communicating with foreign-language patients and working with interpreters and demonstrated improved quality of communication as perceived by patients [23]. Koff et al. developed a medical Spanish website for use in the ED, utilizing phrases and sound clips to facilitate history taking [19]. Mazor et al. demonstrated that a 10week Spanish course for pediatric emergency physicians was associated with decreased interpreter use and increased family satisfaction [24].

Brief language instruction is associated with hazards as well as benefits. An intervention in the USA assessed eight PGY1 emergency medicine residents who underwent a 45$\mathrm{h}$ medical Spanish course in their first residency month. Upon completion, major errors, such as misunderstanding of duration of symptoms and misunderstanding of vocabulary, were found in $14 \%$ of 34 subsequent physicianpatient interactions by these residents over 6 months. The authors caution that although medical language courses may be a useful adjunct to interpreters, they are not designed to replace them, and that significant errors may occur when participants in such courses assume their knowledge is sufficient to obtain a good history, give patient release instructions, and provide medical care in general without an interpreter present [25].

Pronunciation of even short Portuguese phrases is difficult to master and requires practice by clinicians to be successful. Shortening and simplifying several of the phrases may be beneficial. Although the tool employed close-ended questions constructed to elicit meaningful responses to the non-Portuguese speaker, patients would occasionally respond with long narratives that were incomprehensible to the clinician. A possible solution might be to design questions even more strictly close-ended than they presently are and to make available to the clinician the translated phrase "Please answer with only yes or no, or a number."

Limitations of the study include small sample size and poor staff recruitment. Small sample size in this pilot study related chiefly to an unusually low number of ED patients who met the inclusion criteria, and possibly incomplete recruitment of eligible patients by the triage nurse and ED clinician given time constraints. (A repeat study with a 
revised instrument and larger sample size will allow for more meaningful statistic analysis.) Poor staff compliance with practicing with the audio CD prior to bedside pilot of the instrument limited usefulness of the communication tool, and inclusion of phonetic pronunciation on the printed instrument might have mitigated this weakness. Clinician indication of support for using the tool with future patients may promise greater compliance with practicing with the audio guide and greater success with the instrument after successful use. Having patients translate back the question to a bilingual observer would have further confirmed their comprehension and appropriateness of response.

\section{Conclusion}

Language barriers, patient fear, and apprehension impede optimal care. Attempts at cultural competency may go far in enhancing the patient's positive impression of the encounter with the clinician, reducing patient stress, and helping the clinician to more easily elicit patient concerns. Improving language concordance even by learning a few key phrases is likely to have a positive impact on clinical care and demonstrates concern and respect for the patient.

\section{Conflicts of interest None.}

\section{References}

1. Williams MV, Parker R, Baker D et al (1995) Inadequate functional health literacy among patients at two public hospitals. JAMA 274:1677-1682

2. David RA, Rhee M (1998) The impact of language as a barrier to effective health care in an underserved urban Hispanic community. Mt Sinai J Med 65(5-6):393-397

3. Gill JM (1994) Nonurgent use of the emergency department: appropriate or not? Ann Emerg Med 24(5):953-957

4. Petersen LA, Burstin HR, O'Neil AC et al (1998) Nonurgent emergency department visits: the effect of having a regular doctor. Med Care 36(8):1249-1255

5. Pane G, Farmer M, Salness K (1991) Health care access problems of medically indigent emergency department walk-in patients. Ann Emerg Med 20(7):730-733

6. Han A (2005) Access to care prior to the ED visit. MSc thesis, Department of Public Health Sciences, University of Alberta

7. Minkovitz C, Grason H, Aliza B et al (1999) Evaluation of the Community Access to Child Health Program. Pediatrics 103(6 Pt 3): 1384-1393

8. Han A, Ospina MB, Blitz S, Strome T, Rowe BH (2007) Patients presenting to the emergency department: the use of other health care services and reasons for presentation. CJEM 9(6):428-434

9. Ozolins L, Hjelm K (2003) Nurses' experiences of problematic situations with migrants in emergency care in Sweden. Clin Eff Nurs 7(2):84-93
10. Bond K, Ospina MB, Blitz S, Afilalo M, Campbell SG, Bullard M, Innes G, Holroyd B, Curry G, Schull M, Rowe BH (2007) Frequency, determinants and impact of overcrowding in emergency departments in Canada: a national survey. Healthc Q 10(4):3240

11. Derlet RW, Richards JR (2002) Emergency department overcrowding in Florida, New York, and Texas. South Med J 95 (8):846-849

12. Flores G, Abreu M, Olivar MA, Kastner B (1998) Access barriers to health care for Latino children. Arch Pediatr Adolesc Med 152:1119-1125

13. Hampers LC, Cha S, Gutglass DJ, Binns HJ, Krug SE (1999) Language barriers and resource utilization in a pediatric emergency department. Pediatrics 103(6 Pt 1):1253-1256

14. Farmer SA, Higginson IJ (2006) Chest pain: patient perceptions and decision-making in a London emergency department. Ann Emerg Med 48(1):77-85

15. Clarke C, Friedman SM, Shi K, Arenovich T, Culligan C (2005) Emergency department discharge instructions comprehension and compliance study. CJEM 7(1):5-11

16. Crane JA (1997) Patient comprehension of doctor-patient communication on discharge from the emergency department. J Emerg Med 15(1):1-7

17. Goldman RD, Amin P, Macpherson A (2006) Language and length of stay in the pediatric emergency department. Pediatr Emerg Care 22(9):640-643

18. Stewart MA (1995) Effective physician-patient communication and health outcomes: a review. CMAJ 152:1423-1433

19. Koff MD, McGowan JJ (1999) MedSpanish: a language tool for the emergency department. Proc AMIA Symp 1999:565569

20. Lee LJ, Batal HA, Maselli JH, Kutner JS (2002) Effect of Spanish interpretation method on patient satisfaction in an urban walk-in clinic. J Gen Intern Med 17:641-646

21. Hornberger J, Itakura H, Wilson S (1997) Bridging language and cultural barriers between physicians and patients. Public Health Rep 112:410-417

22. Toronto Western Hospital. Available via University Health Network website: http://www.uhn.ca/twh/index.htm. Accessed 10 Nov 2006

23. Bischoff A, Perneager TV, Bovier PA, Loutan L, Stalder H (2003) Improving communication between physicians and patients who speak a foreign language. Br J Gen Pract 53:541-546

24. Mazor SS, Hampers LCC, Chande VT, Krug SE (2002) Teaching Spanish to pediatric emergency physicians: effects on patient satisfaction. Arch Pediatr Adolesc Med 156(7):693-695

25. Prince D, Nelson M (1995) Teaching Spanish to emergency medicine residents. Acad Emerg Med 2(1):32-36

Steven M. Friedman is Assistant Professor, Faculty of Medicine at the University of Toronto.He practices emergency medicine at University Health Network, a quaternary careUniversity of Toronto teaching hospital, where he is Director of the emergency medicineresearch program. His research interests relate to barriers to care and health servicedelivery in the emergency department. In 2007, he was named Researcher of theYear by the University of Toronto Department of Family and Community Medicine.He completed his medical training at the University of Toronto, his emergencymedicine fellowship at McMaster University, Ontario, and an M.P.H. at HarvardUniversity, Cambridge, MA. 